\title{
Vulnerabilidade e Declínio Funcional em pessoas idosas da Atenção Primária à Saúde: estudo longitudinal
}

\author{
Vulnerability and Functional Decline in older people in Primary Health Care: a longitudinal study
}

Juliana Fernandes Cabral' (ID Ageo Mário Cândido da Silva² $\mathbb{D}$ Amanda Cristina de Souza Andrade2 (ID Edilene Gianelli Lopes ${ }^{2} \mathbb{D}$ Inês Echenique Mattos $^{3}$ (D)

\section{Resumo}

Objetivo: Avaliar a associação da vulnerabilidade e o declínio funcional para Atividades Instrumentais de Vida Diária (AIVD) em pessoas idosas atendidas em unidades da Atenção Primária à Saúde (APS) no município de Várzea Grande (MT), Brasil. Método: Estudo longitudinal realizado com 304 pessoas idosas, com acompanhamento de 24 meses. A variável de exposição principal, vulnerabilidade, foi mensurada na linha de base utilizando-se o Vulnerable Elders Survey (VES-13). A variável dependente foi "declínio funcional em AIVD”, definido como a diminuição de pelo menos um ponto no score de capacidade funcional, avaliada pela Escala de Lawton e Brody, entre a coleta da linha de base e o follow-up. As associações entre declínio funcional em AIVD e vulnerabilidade, condições de saúde, fatores sociodemográficos, autoavaliação de saúde, estilo de vida e eventos adversos em saúde foram estimadas por meio do Odds Ratio (OR), utilizando regressão logística binária. Resultados: 35,20\% das pessoas idosas apresentaram declínio da capacidade funcional em AIVD. O declínio funcional se associou no modelo final com a interação entre vulnerabilidade e inatividade física (OR=3,12, IC95\%, 1,42-6,86), insatisfação com a vida (OR=2,23, IC95\%, 1,09-4,56) e hospitalização (OR=2,01, IC 95\%, 1,18-3,41). Conclusão: O declínio funcional em AIVD foi maior nas pessoas idosas vulneráveis que estavam inativas fisicamente, naquelas insatisfeitas com a vida e que foram hospitalizadas durante o período de seguimento, sendo importante que essas condições sejam identificadas precocemente, para que ações de prevenção de declínio funcional sejam implementadas, além dos programas de incentivo à prática de atividade física pelas pessoas idosas.

\footnotetext{
Universidade do Estado de Mato Grosso (Unemat), Departamento de Enfermagem, Tangará da Serra, MT, Brasil.

2 Universidade Federal de Mato Grosso (UFMT), Instituto de Saúde Coletiva, Programa de Pós-graduação em Saúde Coletiva. Cuiabá, MT, Brasil.

3 Fundação Oswaldo Cruz (FIOCRUZ), Escola Nacional de Saúde Pública, Departamento de Epidemiologia. Rio de Janeiro, RJ, Brasil.
}

Palavras-chave: Saúde do Idoso. Fragilidade. Estudos Longitudinais. Atenção Primária à Saúde. 


\section{Abstract}

Objective: To assess the association between vulnerability and functional decline for Instrumental Activities of Daily Living (IADL) in older people treated in Primary Health Care (PHC) units in the municipality of Várzea Grande (MT), Brazil. Method: A longitudinal study was carried out with 304 older people with a 24-month follow-up. The main exposure variable vulnerability was measured at baseline using the Vulnerable Elders Survey (VES-13). The dependent variable was "functional decline in IADL" defined as the decrease of at least one point in the score of functional capacity assessed by the Lawton and Brody Scale between baseline evaluation and the end of follow-up. The associations between the functional decline in IADL and vulnerability, health conditions, sociodemographic factors, self-rated health, lifestyle, and adverse health events were estimated using the Odds Ratio (OR) with binary logistic regression. Results: A decline in functional capacity in IADL was observed in $35,20 \%$ of the cohort members. In the final model, functional decline was associated with the interaction between vulnerability and physical inactivity $(\mathrm{OR}=3.12,95 \% \mathrm{CI}, 1.42-6.86)$, dissatisfaction with life (OR = 2.23, 95\%CI, 1.09-4.56), and hospitalization (OR = 2.01, 95\%CI, 1.18-3.41). Conclusion: Functional decline in IADL was greater in vulnerable older people who were physically inactive, in those dissatisfied with life, and those who were hospitalized during the follow-up period. These conditions must be identified early so that actions to prevent functional decline could be implemented in addition to programs to encourage older people to exercise.

\section{INTRODUÇÃO}

A capacidade funcional constitui importante indicador do grau de independência da pessoa idosa. Geralmente em estudos de base populacional, a capacidade funcional é avaliada por meio da capacidade para realizar Atividades Básicas da Vida Diária (AVD) e Atividades Instrumentais de Vida Diária (AIVD), sendo respectivamente o Índice de Katz e a Escala de Lawton e Brody os instrumentos mais utilizados nas referidas avaliações'.

Os estudos nacionais sobre a temática são em sua maioria transversais ${ }^{2-8}$, porém, são necessários estudos longitudinais que possam avaliar a mudança na capacidade funcional da pessoa idosa ao longo do tempo, em busca de uma longevidade com maior independência, autonomia e qualidade de vida?

No Brasil, foram identificados poucos estudos longitudinais com pessoas idosas da comunidade que avaliaram os fatores de risco para o declínio funcional em atividade básicas e/ou instrumentais de vida diária ${ }^{10-12}$, dentre os riscos identificados estão: ter 80 anos ou mais, baixa escolaridade, sem atividade profissional, inatividade física, não ter companheiro, apresentar sintomas depressivos e fazer uso de psicofármacos.

Apesar de o declínio funcional estar ligado na maioria das vezes ao processo de envelhecimento, ele não pode ser atribuído ao envelhecimento normal, mas às incapacidades mais frequentes na pessoa idosa: incapacidade cognitiva, instabilidade postural, imobilidade, incontinência, incapacidade comunicativa e iatrogenia ${ }^{13}$. Essas incapacidades são preditoras de mortalidade, hospitalização e institucionalização em pessoas idosas ${ }^{13}$. Baseado nessa premissa, foi desenvolvido o Vulnerable Elders Survey (VES-13) ${ }^{14}$, um instrumento simples e eficaz na identificação da pessoa idosa vulnerável, definido como a pessoa idosa que tem risco aumentado de declínio funcional ou morte num período de dois anos.

O VES-13 é um instrumento recomendado pelo Ministério da Saúde (MS) na avaliação da pessoa idosa, que consta na Caderneta de Saúde da Pessoa Idosa ${ }^{15}$ e traz como vantagem o fato de ser curto e de fácil aplicação ${ }^{14}$. No Brasil, a versão original do VES-1 $13^{14}$ sofreu o processo de adaptação transcultural ${ }^{16}$ e validação ${ }^{17}$. Apesar da condição de vulnerabilidade predizer eventos adversos em 
saúde, dentre eles o declínio funcional, nenhum dos estudos supracitados ${ }^{10-12}$, avaliou a associação da vulnerabilidade física e o declínio funcional, nem foram encontrados estudos longitudinais no contexto brasileiro com seguimento superior a seis meses que estudassem essa associação.

Considerando-se o uso do instrumento VES-13 preconizado pelo MS, e mediante a importância do acompanhamento de pessoas idosas vulneráveis em relação aos desfechos adversos em saúde, o estudo objetivou avaliar a associação da vulnerabilidade e o declínio funcional para Atividades Instrumentais de Vida Diária (AIVD) de pessoas idosas atendidas em unidades da Atenção Primária à Saúde (APS) no município de Várzea Grande (MT).

\section{MÉTODO}

Estudo longitudinal, com acompanhamento de 24 meses de pessoas idosas cadastradas nas unidades da APS do município de Várzea Grande, MT, Brasil. A coleta de dados da linha de base ocorreu no período de março a junho de 2016 e o follow-up no período de julho a outubro de 2018. Várzea Grande (MT) é a segunda maior cidade do Estado, com população estimada para 2020 de 287.526 habitantes $^{18}$. No ano de 2016, o município contava com 15 unidades da APS e destas, 11 foram selecionadas para compor a amostra de estudo, por serem campo de estágio curricular de saúde coletiva.

$\mathrm{Na}$ linha de base foi adotado uma amostragem por conglomerados em dois estágios: I) unidades da APS; II) pessoas idosas selecionadas proporcionalmente ao tamanho da população de pessoas de 60 anos ou mais cadastradas em cada unidade. O tamanho da amostra seguiu os procedimentos propostos para populações finitas, utilizando nível de confiança de 0,95, erro tolerável de amostragem de 0,05 e prevalência assumida de vulnerabilidade 0,50 , foram acrescidos $10 \%$ para compensar possíveis perdas, totalizando 377 pessoas idosas. Maiores detalhes sobre a amostragem e coleta de dados da linha de base podem ser consultados na publicação do estudo inicial ${ }^{19}$.

Das 377 pessoas idosas da linha de base, 304 participaram do follow-up. As perdas do follow-up foram devido a não localização da pessoa idosa após 3 visitas em horários diferentes ao domicílio e contato telefônico sem sucesso $(n=49)$ e óbitos $(n=24)$ durante o período de seguimento. Os dados confirmatórios referentes aos óbitos foram obtidos através dos registros do Sistema de Informação de Mortalidade (SIM), disponibilizado pela Secretaria Municipal de Saúde de Várzea Grande.

Para testar o poder da amostra do follow-up $(\mathrm{n}=304)$ foi realizado um teste post-hoc, considerando Odds Ratio de 2,3, proporção de exposição de 0,5 e 0,3 entre os grupos de comparação e o nível de significância de 0,05 , a amostra de estudo apresentou poder de $91,2 \%$.

Os critérios de exclusão do estudo na linha de base ${ }^{19}$ foram: pessoas idosas que apresentaram comprometimento cognitivo após a aplicação do Miniexame do Estado Mental (MEEM) e casos de grave comprometimento da visão e audição, ou sequela grave de Acidente Vascular Encefálico (AVE), que impedissem a pessoa idosa de responder ao questionário. Nos casos de recusa, quando a pessoa idosa não se encontrava em casa no momento da entrevista ou quando apresentava deficit cognitivo, ela era substituída pela pessoa idosa residente mais próxima, que também estivesse cadastrada nas unidades da APS.

As entrevistas ocorreram no próprio domicílio da pessoa idosa, realizadas por entrevistadores treinados, auxiliados pelo Manual do Entrevistador, após realização de estudo piloto e processo de calibração. $\mathrm{Na}$ linha de base as pessoas idosas eram acessadas a partir da visita do Agente Comunitário de Saúde e eram convidadas a participar voluntariamente da pesquisa e em caso de aceite, a entrevista era realizada pelo entrevistador, caso não fosse possível realizar no mesmo dia, um agendamento era feito. No followup a partir da lista de endereços e da identificação das pessoas idosas participantes da linha de base, os entrevistadores visitavam seus domicílios e as convidavam a participarem novamente da pesquisa.

A variável resposta do estudo foi avaliada pela capacidade funcional em AIVD medida na linha de base e no follow-up pela Escala de Lawton e Brody adaptada para população brasileira ${ }^{20}$. O declínio funcional (sim, não) foi definido como 
a diminuição de pelo menos 1 ponto no score da capacidade funcional em AIVD entre a coleta da linha de base e o follow-up, independentemente do grau de dependência da pessoa idosa na linha de base ${ }^{21}$.

A escala avalia oito atividades, como utilizar o telefone, utilizar meios de transporte, fazer compras, arrumar a casa, preparar refeições, lavar a roupa, controlar o dinheiro e tomar medicamentos. Cada questão tem três possibilidades de resposta, e cada resposta gera uma pontuação de 1 a 3: 1 ponto para quem não executa a referida atividade (dependente); 2 pontos, para quem executa a atividade com auxílio (dependência parcial) e 3 pontos para quem executa a atividade sem auxílio (independente). A Pontuação final (score) é a somatória dos oito domínios e pode variar de 8 a 24 pontos, quanto maior for o score, mais independente é aquele indivíduo ${ }^{21}$.

A variável de exposição principal do estudo foi a vulnerabilidade (sim; não), mensurada somente na linha de base pelo instrumento Vulnerable Elders Survey (VES-13), adaptado e validado para uso na população brasileira $^{16,17}$. O instrumento é composto por 13 itens que contemplam: idade, saúde autorreferida, capacidade física e capacidade funcional e seu score varia entre 0 e 13 pontos, sendo a pontuação igual ou maior que três $(3,0)$ considerada como ponto de corte para classificar o indivíduo como vulnerável ${ }^{14}$.

As covariáveis relacionadas às condições de saúde foram avaliadas na linha de base e estão explicitadas no estudo inicia ${ }^{19}$ : A Escala de Depressão Geriátrica 15 (GDS-15) $^{22}$ utilizada para avaliação de sintomas depressivos (score $\leq 5$ pontos, sem sintomatologia depressiva; e score $\geq 6$ pontos, com sintomatologia depressiva), a Mini Avalição Nutricional Reduzida $(\mathrm{MANR})^{23}$ para avaliação do estado nutricional. A soma da pontuação obtida em cada item da MANR é utilizada para classificar os indivíduos em três categorias (desnutrido: 0 a 7 pontos; em risco de desnutrição: 8 a 11 pontos e estado nutricional normal: 12 a 14 pontos), no presente estudo unimos as categorias desnutrido e em risco nutricional. $\mathrm{O}$ Cumulative Illness Rating Scale - Geriatric (CIRS-G) ${ }^{24}$, para avaliação da comorbidade (sem comorbidade de nível de gravidade 3 ou 4; com comorbidade de nível de gravidade 3 ou 4), a fragilidade (sim: $\geq 5$ pontos; não: $<5$ pontos), avaliada através do instrumento
Tilburg Frailty Indicator (TFI ${ }^{25}$, além da polifarmácia (sim; não), considerada como o uso contínuo de 5 ou mais medicamentos.

Outras covariáveis do estudo foram as condições sociodemográficas, de autoavaliação de saúde, estilo de vida e eventos adversos em saúde, coletadas no follow-up. Sociodemográficas: a) sexo (masculino; feminino); b) faixa etária (60 a 69 anos; 70 anos e mais); c) estado conjugal (vive sem companheiro(a), vive com companheiro(a)); d) escolaridade (não alfabetizado(a); alfabetizado(a)), renda per capita (até $1 / 2$ SalárioMínimo; >1/2 Salário-Mínimo). Autoavaliação de saúde: avaliação positiva (autorreferiram saúde boa ou muito boa) e avaliação negativa (autorreferiram saúde regular, ruim ou muito ruim). Estilo de vida: a) satisfação com a vida (não; sim); b) hábito de fumar (sim, para aqueles que fumam atualmente ou já fumaram; não, para aqueles que nunca tiveram o hábito tabágico); c) hábito de ingerir álcool (sim, para aqueles que ingerem álcool atualmente ou já tiveram esse hábito; não, para aqueles que nunca tiveram o hábito de ingerir álcool); d) prática regular de atividade física nos 12 meses anteriores à coleta de dados (não; sim). Eventos adversos em saúde: a) referir ao menos uma morbidade (sim; não); b) adoecimento grave nos últimos 12 meses anteriores à coleta de dados (sim; não); c) hospitalização, quedas, e fraturas nos últimos 24 meses anteriores à coleta de dados (sim; não).

Foram calculados os Odds Ratio (OR) (com IC 95\% para OR) como medida de associação entre a variável dependente "declínio funcional" e as variáveis vulnerabilidade, condições de saúde, sociodemográficas, autoavaliação de saúde, estilo de vida e eventos adversos em saúde, estimada por meio de Regressão Logística. As covariáveis que apresentaram $p$-valor $<0,20$ na análise bivariada foram incluídas na análise múltipla. Foi utilizado o modelo stepwise backward, onde foram retiradas progressivamente do modelo as variáveis que não se apresentaram estatisticamente significantes, sendo mantidas no modelo final as varáveis com $p$-valor $<0,05$. A variável de exposição principal "vulnerabilidade" e as variáveis de ajuste "sexo" e "faixa etária" foram mantidas no modelo múltiplo independente da significância estatística. Foram testadas as interações entre a vulnerabilidade e as covariáveis do modelo final. 
Este estudo foi submetido e aprovado pelo Comitê de Ética em Pesquisa da Universidade do Estado de Mato Grosso sob número de parecer 2.771.193. e seguiu todas as recomendações constantes nas Resoluções 466/2012, 510/2016 e 580/2018 do Conselho Nacional de Saúde.

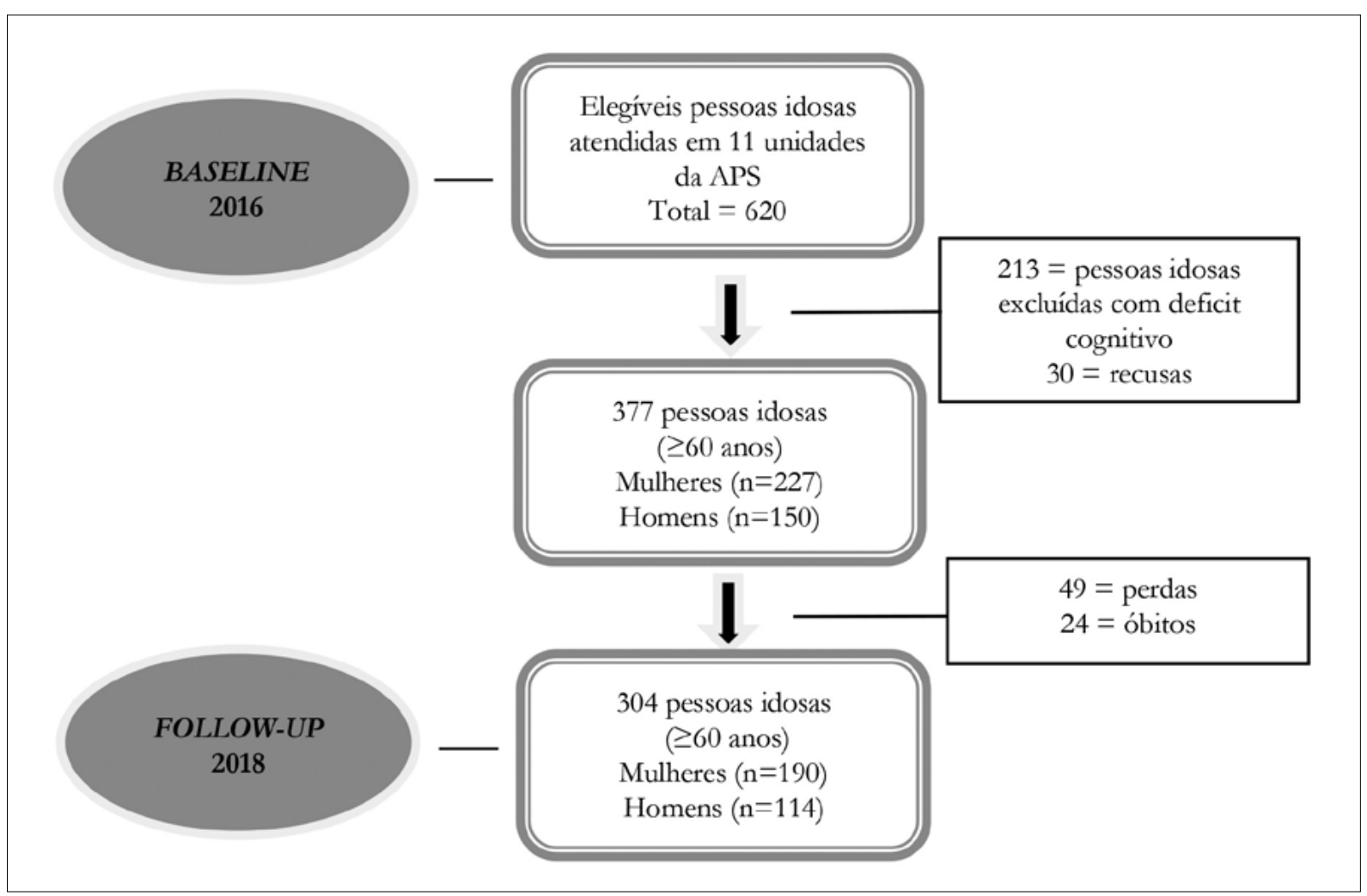

Figura 1. Fluxograma de pessoas idosas participantes do estudo longitudinal (2016/2018). Várzea Grande (MT), 2020.

\section{RESULTADOS}

Participaram do estudo 304 pessoas idosas, onde na linha de base $62,30 \%$ das pessoas idosas eram dependentes para as AIVD e 49,07\% eram vulneráveis. No follow-up, 62,50\% apresentaram dependência nas AIVD. O declínio da capacidade funcional em AIVD foi de 35,20\%. Quanto ao perfil sociodemográfico, a maioria foi do sexo feminino $(62,50 \%)$, na faixa etária de 70 anos e mais $(53,29 \%)$, média de 71,79 anos, com mediana de 70 anos $(\mathrm{DP} \pm 7,42)$; se autodeclararam pretos e pardos (78,62\%); possuíam companheiro (casado ou união estável) (55,92\%); cursaram o primeiro grau $(66,12 \%)$ e tinham renda mensal per capita $>1 / 2$ salário-mínimo $(53,95 \%)$.
$\mathrm{Na}$ análise bruta das variáveis sociodemográficas, autoavaliação de saúde e estilo de vida, observou-se declínio funcional nas pessoas idosas que referiram estarem insatisfeitas com a vida (OR=2,43, IC95\%, $1,21-4,88)$ e que não realizavam atividade física $(\mathrm{OR}=2,50, \mathrm{IC} 95 \%, 1,40-4,44)$ (Tabela 1).

$\mathrm{Na}$ análise bruta das variáveis relacionadas às condições de saúde, nenhuma das variáveis apresentou associação estatisticamente significante (Tabela 2).

$\mathrm{Na}$ análise bruta das variáveis relacionadas aos eventos adversos em saúde, observou-se declínio funcional nas pessoas idosas que tiveram algum adoecimento grave durante o seguimento $(\mathrm{OR}=2,10$, IC95\%, 1,23-3,61), foram hospitalizadas (OR=1,96, 
IC95\%, 1,17-3,27), sofreram quedas $(\mathrm{OR}=1,67$, funcional no modelo final foram a interação entre IC95\%, 1,03-2,71) e fraturas (OR=2,80, IC95\%, a vulnerabilidade e a inatividade física $(\mathrm{OR}=3,12$, 1,03-7,58) (Tabela 3).

As variáveis que permaneceram com associação IC95\%, 1,42-6,86), estar insatisfeito com a vida $(\mathrm{OR}=2,23$, IC95\%, 1,09-4,56) e ter sido hospitalizado nos 24 meses anteriores à coleta de dados do follow-up estatisticamente significante com o declínio $(\mathrm{OR}=2,01$, IC95\%, 1,18-3,41) (Tabela 4).

Tabela 1. Declínio funcional em Atividades Instrumentais de Vida Diária de pessoas idosas segundo variáveis sociodemográficas, autoavaliação de saúde e estilo de vida, medidas no follow-up. Várzea Grande, MT, Brasil, 2020.

\begin{tabular}{|c|c|c|c|c|c|}
\hline \multirow[t]{2}{*}{ Variáveis } & \multicolumn{5}{|c|}{ Declínio funcional } \\
\hline & $\mathrm{n} / \mathrm{N}$ & $\%$ & OR & IC95\% & $p$ - valor \\
\hline \multicolumn{6}{|l|}{ Sexo } \\
\hline Masculino & $38 / 112$ & 33,93 & 1 & \multirow{2}{*}{$0,67-1,78$} & \multirow{2}{*}{0,724} \\
\hline Feminino & $69 / 192$ & 35,94 & 1,09 & & \\
\hline \multicolumn{6}{|l|}{ Faixa etária (em anos) } \\
\hline 60 a 69 & $56 / 167$ & 33,53 & 1 & \multirow{2}{*}{$0,73-1,89$} & \multirow{2}{*}{0,502} \\
\hline 70 e mais & $51 / 137$ & 37,23 & 1,18 & & \\
\hline \multicolumn{6}{|l|}{ Estado conjugal } \\
\hline Vive com companheiro (a) & $62 / 170$ & 36,47 & 1 & \multirow{2}{*}{$0,55-1,42$} & \multirow{2}{*}{0,601} \\
\hline Vive sem companheiro (a) & $45 / 134$ & 33,58 & 0,88 & & \\
\hline \multicolumn{6}{|l|}{ Escolaridade } \\
\hline Alfabetizado (a) & $68 / 195$ & 34,87 & 1 & \multirow{2}{*}{$0,64-1,70$} & \multirow{2}{*}{0,874} \\
\hline Não alfabetizado (a) & $39 / 109$ & 35,78 & 1,04 & & \\
\hline \multicolumn{6}{|l|}{ Renda per capita (em SM) } \\
\hline Até $1 \mathrm{SM}$ & $34 / 96$ & 35,42 & 1 & \multirow{2}{*}{$0,59-1,64$} & \multirow{2}{*}{0,957} \\
\hline$\geq 1 \mathrm{SM}$ & $73 / 208$ & 35,10 & 0,99 & & \\
\hline \multicolumn{6}{|l|}{ Autoavaliação de saúde } \\
\hline Avaliação positiva & $26 / 95$ & 27,37 & 1 & \multirow{2}{*}{$0,99-2,85$} & \multirow{2}{*}{0,055} \\
\hline Avaliação negativa & $81 / 209$ & 38,76 & 1,68 & & \\
\hline \multicolumn{6}{|l|}{ Satisfação com a vida } \\
\hline $\operatorname{Sim}$ & $87 / 267$ & 32,58 & 1 & \multirow{2}{*}{$1,21-4,88$} & \multirow{2}{*}{0,012} \\
\hline Não & $20 / 37$ & 54,05 & 2,43 & & \\
\hline \multicolumn{6}{|l|}{ Hábito de fumar } \\
\hline Não & $48 / 147$ & 32,65 & 1 & \multirow{2}{*}{$0,76-1,96$} & \multirow{2}{*}{0,409} \\
\hline Sim & $58 / 157$ & 37,18 & 1,22 & & \\
\hline \multicolumn{6}{|l|}{ Hábito de ingerir álcool } \\
\hline Não & $53 / 145$ & 36,55 & 1 & \multirow{2}{*}{$0,56-1,43$} & \multirow{2}{*}{0,637} \\
\hline $\operatorname{Sim}$ & $54 / 159$ & 33,96 & 0,89 & & \\
\hline \multicolumn{6}{|l|}{ Atividade Física } \\
\hline $\operatorname{Sim}$ & $19 / 88$ & 21,59 & 1 & 140444 & 0002 \\
\hline Não & $88 / 216$ & 40,74 & 2,50 & $1,40-4,44$ & 0,002 \\
\hline
\end{tabular}

OR: Odds Ratio; IC95\%: Intervalo de Confiança de 95\%; SM (Salário-Mínimo): R\$ 954 reais. 
Tabela 2. Declínio funcional em Atividades Instrumentais de Vida Diária de pessoas idosas segundo as variáveis de condições de saúde medidas na linha de base. Várzea Grande, MT, Brasil, 2020.

\begin{tabular}{|c|c|c|c|c|c|}
\hline \multirow{2}{*}{ Variáveis } & \multicolumn{5}{|c|}{ Declínio funcional } \\
\hline & $\mathrm{n} / \mathrm{N}$ & $\%$ & OR & IC95\% & $p$ - valor \\
\hline \multicolumn{6}{|l|}{ Vulnerabilidade (VES-13) } \\
\hline Não vulnerável & $49 / 156$ & 31,41 & 1 & \multirow{2}{*}{$0,88-2,26$} & \multirow{2}{*}{0,156} \\
\hline Vulnerável & $58 / 148$ & 39,19 & 1,41 & & \\
\hline \multicolumn{6}{|c|}{ Comorbidade nível 3 ou 4 (CIRS-G) } \\
\hline Não & $66 / 183$ & 36,07 & 1 & \multirow{2}{*}{$0,56-1,47$} & \multirow{2}{*}{0,697} \\
\hline Sim & $41 / 121$ & 33,88 & 0,91 & & \\
\hline \multicolumn{6}{|l|}{ Nutrição (MANR) } \\
\hline Sem risco nutricional & $57 / 166$ & 34,34 & 1 & \multirow{2}{*}{$0,68-1,74$} & \multirow{2}{*}{0,731} \\
\hline Desnutrição e em risco & $50 / 138$ & 36,23 & 1,09 & & \\
\hline \multicolumn{6}{|l|}{ Depressão (GDS-15) } \\
\hline Sem sintomas depressivos & $74 / 212$ & 34,91 & 1 & \multirow{2}{*}{$0,63-1,74$} & \multirow{2}{*}{0,872} \\
\hline Com sintomas depressivos & $33 / 92$ & 35,87 & 1,04 & & \\
\hline \multicolumn{6}{|l|}{ Fragilidade (TFI) } \\
\hline Não frágil & $38 / 105$ & 36,19 & 1 & \multirow{2}{*}{$0,57-1,53$} & \multirow{2}{*}{0,792} \\
\hline Frágil & 69/199 & 34,67 & 0,94 & & \\
\hline \multicolumn{6}{|l|}{ Polifarmácia* } \\
\hline Não & $76 / 218$ & 34,86 & 1 & \multirow{2}{*}{$0,60-1,89$} & \multirow{2}{*}{0,823} \\
\hline Sim & $24 / 66$ & 36,36 & 1,07 & & \\
\hline
\end{tabular}

OR: Odds Ratio; IC $95 \%$ : Intervalo de Confiança de $95 \%{ }^{*}$ Uso de $\geq 5$ medicamentos.

Tabela 3. Declínio funcional em Atividades Instrumentais de Vida Diária de pessoas idosas segundo as variáveis eventos adversos em saúde, medidas no follow-up. Várzea Grande, MT, Brasil, 2020.

\begin{tabular}{|c|c|c|c|c|c|}
\hline \multirow{2}{*}{ Variáveis } & \multicolumn{5}{|c|}{ Declínio funcional } \\
\hline & $\mathrm{n} / \mathrm{N}$ & $\%$ & OR & IC95\% & $p$ - valor \\
\hline \multicolumn{6}{|c|}{ Refere ao menos uma morbidade } \\
\hline Não & $2 / 11$ & 18,18 & 1 & $0,53-11,85$ & 0,244 \\
\hline Sim & $105 / 293$ & 35,84 & 2,51 & & \\
\hline \multicolumn{6}{|c|}{ Adoecimento grave } \\
\hline Não & $72 / 232$ & 31,03 & 1 & $1,23-3,61$ & 0,007 \\
\hline Sim & $35 / 72$ & 48,61 & 2,10 & & \\
\hline \multicolumn{6}{|c|}{ Hospitalização } \\
\hline Não & $67 / 218$ & 30,73 & 1 & $1,17-3,27$ & 0,010 \\
\hline Sim & $40 / 86$ & 46,51 & 1,96 & & \\
\hline \multicolumn{6}{|l|}{ Quedas } \\
\hline Não & $60 / 194$ & 30,93 & 1 & $1,03-2,71$ & 0,039 \\
\hline Sim & $47 / 110$ & 42,73 & 1,67 & & \\
\hline \multicolumn{6}{|l|}{ Fraturas } \\
\hline Não & $97 / 287$ & 33,80 & 1 & $1,03-7,58$ & 0,043 \\
\hline $\operatorname{Sim}$ & $10 / 17$ & 58,82 & 2,80 & & \\
\hline
\end{tabular}

OR: Odds Ratio; IC95\%: Intervalo de Confiança de 95\%. 
Tabela 4. Resultados da Regressão Logística ajustada entre as variáveis independentes e o declínio funcional (variável dependente). Várzea Grande, MT, Brasil, 2020.

\begin{tabular}{|c|c|c|c|c|}
\hline \multicolumn{2}{|l|}{ Variáveis } & OR Ajustado & $\mathrm{IC}(95 \%)$ & $p$-valor \\
\hline \multicolumn{5}{|l|}{ Sexo } \\
\hline \multicolumn{2}{|l|}{ Masculino } & 1 & $0,60-1,62$ & 0,857 \\
\hline \multicolumn{2}{|l|}{ Feminino } & 0,95 & & \\
\hline \multicolumn{5}{|c|}{ Faixa etária (em anos) } \\
\hline \multicolumn{2}{|c|}{60 a 69} & 1 & $0,73-1,97$ & 0,481 \\
\hline \multicolumn{2}{|l|}{70 e mais } & 1,20 & & \\
\hline \multicolumn{5}{|c|}{ Interação Vulnerabilidade e Atividade física } \\
\hline Não-vulnerável & Ativo & 1 & & \\
\hline Não-Vulnerável & Inativo & 2,22 & $0,99-4,90$ & 0,049 \\
\hline Vulnerável & Ativo & 1,23 & $0,43-3,54$ & 0,695 \\
\hline Vulnerável & Inativo & 3,12 & $1,42-6,86$ & 0,005 \\
\hline \multicolumn{5}{|c|}{ Satisfação com a vida } \\
\hline \multicolumn{2}{|l|}{$\operatorname{Sim}$} & 1 & $1,09-4,56$ & 0,029 \\
\hline \multicolumn{2}{|l|}{ Não } & 2,23 & & \\
\hline \multicolumn{5}{|l|}{ Hospitalização } \\
\hline \multicolumn{2}{|l|}{ Não } & 1 & $1,18-3,41$ & 0,010 \\
\hline \multicolumn{2}{|l|}{$\operatorname{Sim}$} & 2,01 & & \\
\hline
\end{tabular}

OR: Odds Ratio; IC95\%: Intervalo de Confiança de 95\%.

\section{DISCUSSÃO}

O presente estudo é do tipo longitudinal com acompanhamento de 24 meses de pessoas idosas cadastradas nas unidades da APS do município de Várzea Grande (MT), o qual verificou associação positiva entre declínio funcional e interação entre vulnerabilidade e inatividade física, insatisfação com a vida e hospitalização nos 24 meses anteriores à coleta de dados do follow-up.

A proporção de declínio funcional entre pessoas idosas pesquisadas foi elevada ao se comparar com outros estudos realizados com pessoas idosas da comunidade e que também utilizaram a escala de Lawton e Brody na avaliação ${ }^{5,6}$. Entretanto, a comparação com outros estudos é dificultada pela variedade de escalas de aferição de capacidade funcional utilizadas, diferentes definições de declínio funcional, tipos de estudo e as populações-alvo. No Brasil, ainda são escassos os estudos longitudinais sobre o declínio funcional nas AIVD, especialmente em pessoas idosas da comunidade ${ }^{11}$.
Estudo longitudinal ${ }^{26}$ realizado com pessoas idosas brasileiras institucionalizadas residentes em Lares para pessoas idosas do município de Natal ( $\mathrm{RN}$ ), que verificou a probabilidade de manutenção da capacidade funcional nas Atividades Básicas de Vida Diária (AVD), encontrou incidência de declínio funcional de $54 \%$ no período de dois anos. No estudo $^{11}$ longitudinal realizado em Lafaiete Coutinho (BA), com pessoas idosas residentes na comunidade, inicialmente independentes para AVD, a incidência de declínio funcional foi de $15,3 \%$, num seguimento de três anos. Em estudo ${ }^{12}$ longitudinal de base populacional, com dados da coorte de pessoas idosas do Projeto Bambuí (MG) entre os anos de 1998 e 2011, com pessoas idosas independentes para AIVD e AVD na linha de base, a incidência de declínio funcional para as AIVD observada foi de $58 \%$ e para as AVD foi de $44,5 \%$. Os resultados discrepantes da incidência de declínio funcional para as AVD, provavelmente se deu em decorrência de a população-alvo do primeiro ${ }^{26}$ estudo serem de pessoas idosas institucionalizadas, mais vulneráveis fisicamente, em relação as pessoas 
idosas da comunidade do segundo estudo ${ }^{11}$ e pelo maior período de seguimento da coorte de Bambuí ${ }^{12}$.

A vulnerabilidade se associou ao declínio funcional entre pessoas idosas inativas. Em uma coorte $^{27}$, que acompanhou 635 pessoas idosas da Atenção Primária em Saúde na cidade do Rio de Janeiro (RJ), utilizando o VES-13 para avaliar a vulnerabilidade e a Escala de Lawton e Brody para avaliação de capacidade funcional em AIVD, identificou que o declínio funcional num período de seis meses, foi maior entre os indivíduos vulneráveis (OR=1,95; CI $95 \%$ 1,49-2,54).

A condição de vulnerabilidade da pessoa idosa e inatividade física representou maior risco para o desenvolvimento de declínio funcional em AIVD. A associação pode ser explicada pelo fato de que a inatividade física agrava a condição de vulnerabilidade, fazendo com que a pessoa idosa deixe de melhorar sua aptidão cardiorrespiratória e muscular, a saúde óssea e funcional ${ }^{28}$. A Organização Mundial da Saúde (OMS) estimula a realização de atividade física, que inclui atividade recreativa ou de lazer, transporte (caminhada ou ciclismo), ocupacional (se a pessoa idosa ainda trabalhar), tarefas domésticas, esportes ou exercícios planejados, no âmbito de atividades diárias, familiares e comunitárias ${ }^{28}$.

Quanto maior a frequência de atividades físicas pela pessoa idosa, menor o risco para o desenvolvimento de incapacidades em AVD e AIVD $^{28,29}$. Conforme as novas Diretrizes da OMS (2020) sobre atividade física e comportamento sedentário, resultado de uma abrangente revisão sistemática, há uma forte evidência de uma relação dose-resposta inversa entre o volume de atividade física aeróbia e o risco de limitações físicas funcionais em pessoas idosas ${ }^{28}$.

Pesquisa de base populacional, que acompanhou múltiplas coortes e avaliou os fatores de risco para a perda da funcionalidade em pessoas idosas, concluiu que as pessoas idosas com baixo nível socioeconômico e presença de fatores de risco como doenças crônicas, inatividade física, alto consumo de álcool e tabaco, além da obesidade, tiveram maior perda de capacidade funcional ${ }^{9}$.
O acompanhamento de duas coortes de pessoas idosas da comunidade, uma com 403 pessoas idosas italianas e outra com 395 pessoas idosas holandesas, na faixa etária de 60 a 70 anos, que avaliaram o declínio funcional num seguimento de 9 anos, utilizando alguns itens das AVD e AIVD, a maioria das pessoas idosas referiu não ter declínio funcional na linha de base ${ }^{30}$. Os preditores para limitações funcionais nos homens foram o medo de cair e o consumo de álcool, já no sexo feminino os preditores foram idade, atividade física, morar sozinha, satisfação econômica, velocidade da marcha, Índice de Massa Corporal e doenças cardiovasculares ${ }^{30}$.

Estudos realizados com pessoas idosas brasileiras residentes na comunidade encontraram associação entre menor nível de atividade física e a incapacidade funcional nas $\mathrm{AIVD}^{7,10}$. Contudo, nem sempre a inatividade física isoladamente explica a incapacidade, como evidenciado no estudo longitudinal ${ }^{11}$ de um município baiano com pessoas idosas não institucionalizadas, onde não foi encontrada associação entre declínio funcional e realização insuficiente de atividade física.

O fato de a pessoa idosa estar insatisfeita com a vida também se associou ao declínio funcional, o que corrobora com os achados de Nunes et al..$^{5}$ onde o fato de a pessoa idosa estar mais satisfeita com a vida implica em menor prevalência de incapacidade funcional. Essa associação positiva entre a maior satisfação com a vida e a independência nas AIVD justifica-se pelo fato de que a funcionalidade está relacionada à melhor manutenção da saúde e qualidade de vida da pessoa idosa e, portanto, maior satisfação com a vida no envelhecimento ${ }^{31}$.

Estudo que avaliou a depressão em pessoas idosas da comunidade no sul do Brasil encontrou forte associação com insatisfação com a vida, o que indica que essa variável é uma boa marcadora para rastrear sintomas depressivos em pessoas idosas ${ }^{8}$. Por sua vez, boas condições econômicas, alta escolaridade, ausência de incapacidades físicas, autoavaliação positiva de saúde, capacidade cognitiva e acesso aos serviços de saúde são aspectos que explicam maiores níveis de satisfação com a vida ${ }^{32}$. A incapacidade funcional em AVD e AIVD também se associou 
ao uso de benzodiazepínicos, antidepressivos e antipsicóticos, medicamentos utilizados para transtornos de humor e comportamentais ${ }^{12}$.

A ocorrência de hospitalização da pessoa idosa no período entre as coletas da linha de base e do follow-up se associou ao declínio funcional nas AIVD. A hospitalização de pessoas idosas leva ao declínio funcional, incapacidade, morbidade e mortalidade $^{33}$. A hospitalização é um risco para a população idosa, apresentando chances aumentadas para o desenvolvimento de eventos adversos, dentre os mais importantes o declínio funcional, sendo que os principais fatores de risco para o declínio funcional associados à hospitalização são: idade, imobilidade, comprometimento cognitivo e status funcional antes da internação ${ }^{34}$. Admi et al. ${ }^{33}$ encontraram discordâncias nos estudos quanto ao momento da ocorrência do declínio funcional, apontando que este pode ocorrer na pré-admissão, na admissão, durante a hospitalização e até mesmo após a alta hospitalar. Uma coorte de pessoas idosas hospitalizadas, evidenciou piora da funcionalidade após a alta hospitalar ${ }^{35}$.

Uma das vantagens do nosso estudo foi o acompanhamento longitudinal dos idosos por 24 meses. Contudo, neste tipo de estudo, a perda de participantes ao longo do seguimento pode representar uma limitação. A realização de entrevistas por equipe treinada pode ter minimizado tanto as perdas quanto os vieses de informação, pela padronização da coleta de dados. Outra provável limitação do presente estudo foi que as informações da capacidade funcional foram autorreferidas e não se pôde avaliar o risco de desenvolver o declínio funcional, pois na linha de base já havia alta proporção de pessoas idosas com incapacidade.

O estudo de seguimento com essa população foi importante para um melhor entendimento das condições que se associam ao declínio funcional em pessoas idosas atendidas nas unidades da APS. Os resultados do presente estudo reforçam a importância de identificar precocemente eventos que causam o declínio funcional da pessoa idosa, intervindo de maneira a evitar que se tornem dependentes.

\section{CONCLUSÃO}

O declínio funcional de pessoas idosas que eram atendidas nas unidades da APS associou-se com a interação entre vulnerabilidade e inatividade física, insatisfação com a vida e hospitalização no período de dois anos de seguimento.

Esses resultados demonstram a importância de se conhecer a condição de vulnerabilidade das pessoas idosas da comunidade, porque ela pode indicar condições adversas em saúde como o declínio funcional, o que pode levar ao isolamento social da pessoa idosa, dependência de cuidados, dependência financeira, limitações físicas, entre outros.

Sugerem-se que estudos longitudinais acompanhem pessoas idosas independentes para as Atividades Instrumentais de Vida Diária na linha de base, por um período maior de seguimento, favorecendo uma avaliação mais ampliada dos desfechos relacionados à vulnerabilidade e seus fatores preditores, além de avaliar o efeito da atividade física na reversão da condição de vulnerabilidade. Ademais, seria importante o desenvolvimento de um plano de atenção gerontológica que pudesse identificar quais são as pessoas idosas vulneráveis residentes na comunidade e, posteriormente, fazer uma avaliação geriátrica multidimensional para investigar as causas e intervir, atuando assim na prevenção do declínio funcional e/ou reversão dele. Além disso, implementar programas de incentivo à prática de atividade física, favorecendo assim a melhoria da capacidade funcional, qualidade de vida e manutenção da autonomia dessas pessoas idosas.

Editado por: Ana Carolina Lima Cavaletti 


\section{REFERÊNCIAS}

1. Veras RP. Guia dos Instrumentos de Avaliação Geriátrica [Internet]. Rio de Janeiro: Unati/UERJ; 2019 [acesso em 17 abr. 2021]. Disponível em: https://www.unatiuerj.com.br/Guia $\% 20 \operatorname{dos} \% 20$ instrumentos $\% 20$ Avaliacao $\% 20$ Geriatrica.pdf.

2. Gavasso WC, Beltrame V. Capacidade funcional e morbidades referidas: uma análise comparativa em idosos. Rev Bras Geriatr Gerontol. 2017;20(3):399409. Disponível em: https://doi.org/10.1590/198122562017020.160080 .

3. Aguiar BM, Silva PO, Vieira MS, Costa FM, Carneiro JA. Avaliação da incapacidade funcional e fatores associados em idosos. Rev Bras Geriatr Gerontol. 2019;22(2):e180163. Disponível em: https://doi. org/10.1590/1981-22562019022.180163.

4. Cardoso JDC, Oliveira AD, Cunha CRT, Silva KM. Capacidade funcional de idosos residentes em zona urbana. Rev Enferm UFSM. 2019;9(19):1-14. Disponível em: https://doi. org/10.5902/2179769234095.

5. Nunes JD, Saes MO, Nunes BP, Siqueira FCV, Soares DC, Fassa MEG, et al. Indicadores de incapacidade funcional e fatores associados em idosos: estudo de base populacional em Bagé, Rio Grande do Sul. Epidemiol Serv Saúde. 2017;26(2):295-304. Disponível em: https://doi.org/10.5123/s167949742017000200007.

6. Pereira LC, Figueiredo MLF, Beleza CMF, Andrade EMLR, Silva MJ, Pereira AFM. Fatores preditores para incapacidade funcional de idosos atendidos na atenção básica. Rev Bras Enferm. 2017;70(1):112-8. Disponível em: https://doi.org/10.1590/0034-71672016-0046.

7. Virtuoso-Júnior JS, Tribess S, Smith Menezes A, Meneguci J, Sasaki JE. Fatores associados à incapacidade funcional em idosos brasileiros. Rev Andal Med Deporte. 2016. Disponível em: https:// doi.org/10.1016/j.ramd.2016.05.003.

8. Bretanha AF, Facchini LA, Nunes BP, Munhoz TN, Tomasi El, Thumé E. Sintomas depressivos em idosos residentes em áreas de abrangência das Unidades Básicas de Saúde da zona urbana de Bagé, RS. Rev Bras Epidemiol. 2015;18(1):1-12. Disponível em: https://doi.org/10.1590/1980-5497201500010001.

9. Stringhini S, Carmeli C, Jokela M, Avendaño M, McCrory C, d'Errico A, et al. Socioeconomic status, non-communicable disease risk factors, and walking speed in older adults: multi-cohort population based study. BMJ. 2018;360:k1046. Disponível em: https:// doi.org/10.1136/bmj.k1046.
10. Ikegami EM, Souza LA, Tavares DMS, Rodrigues LR. Capacidade funcional e desempenho físico de idosos comunitários: um estudo longitudinal. Ciênc Saúde Colet. 2020;25(3):1083-90. Disponível em: https://doi.org/10.1590/1413-81232020253.18512018.

11. Matos FS, Jesus CS, Carneiro JAO, Coqueiro RS, Fernandes MH, Brito TA. Redução da capacidade funcional de idosos residentes em comunidade: estudo longitudinal. Ciênc Saúde Colet. 2018;23(10):3393401. Disponível em: https://doi.org/10.1590/1413812320182310.23382016 .

12. Falci DM, Mambrini JVM, Castro-Costa E, Firmo JOA, Lima-Costa MF, Loyola Filho AI. Uso de psicofármacos prediz incapacidade funcional entre idosos. Rev Saúde Pública. 2019;53(21):1-10. Disponível em: https://doi.org/10.11606/s15188787.2019053000675.

13. Moraes EM. Atenção à saúde do Idoso: aspectos conceituais. Brasília, DF: OPAS; 2012. Disponível em: https://bibliodigital.unijui.edu.br:8443/ xmlui/bitstream/handle/123456789/5574/ aten $\%$ c3 $\%$ a $7 \%$ c3 $\%$ a3o $\% 20$ a $\% 20$ saude $\% 20 \mathrm{do} \% 20$ idoso.pdf? sequence $=1 \&$ is Allowed $=\mathrm{y}$.

14. Saliba D, Elliott M, Rubenstein LZ, Solomon DH, Young RT, Kamberg CJ, et al. The Vulnerable elders survey: a tool for identifying vulnerable older people in the community. J Am Geriatr Soc. 2001;49(12):1691-9. Disponível em: https://doi. org/10.1046/j.1532-5415.2001.49281.x.

15. Brasil. Ministério da Saúde, Secretaria de Atenção à Saúde. Manual para utilização da Caderneta de Saúde da Pessoa Idosa. Brasília, DF: Ministério da Saúde; 2018. Disponível em: http://bvsms.saude.gov.br/bvs/ publicacoes/manual_utilizacao_caderneta_pessoa_ idosa.pdf.

16. Luz LL, Santiago LM, Silva JFS, Mattos IE. Primeira etapa da adaptação transcultural do instrumento The Vulnerable Elders Survey (VES-13) para o Português. Cad Saúde Pública. 2013;29(3):621-8. Disponível em: https://www. scielosp.org/pdf/csp/2013.v29n3/621-628/pt .

17. Luz LL, Santiago LM, Silva JFS, Mattos IE. Psychometric properties of the Brazilian version of the Vulnerable Elders Survey-13 (VES-13). Cad Saúde Pública. 2015;31(3):507-15. Disponível em: https://doi. org/10.1590/0102-311x00011714.

18. Instituto Brasileiro de Geografia e Estatística, Diretoria de Pesquisas, Coordenação de População e Indicadores Sociais. Estimativas da população residente com data de referência $1^{\text {a }}$ de julho de 2020: Várzea Grande: IBGE; 2020. Disponível em: https://cidades.ibge.gov. $\mathrm{br} /$ brasil/mt/varzea-grande/panorama. 
19. Cabral JF, Silva AMC, Mattos IE, Neves AQ, Luz LL, Ferreira DB, et al. Vulnerabilidade e fatores associados em idosos atendidos pela Estratégia Saúde da Família. Ciênc Saúde Colet. 2019;24(9):322736. Disponível em: https://doi.org/10.1590/141381232018249.22962017.

20. Santos RL, Virtuoso-Júnior JS. Confiabilidade da versão brasileira da escala de atividades instrumentais de vida diária. Rev Bras Promoç Saúde. 2008;21(4):290-6. Disponível em: https://doi. org $/ 10.5020 / 575$.

21. Lawton MP, Brody EM. Assessment of older people: self-maintaining and instrumental activities of daily living. Gerontologist. 1969;9:179-86. Disponível em: https://doi.org/10.1093/geront/9.3_Part_1.179.

22. Almeida OP, Almeida SA. Short versions of the geriatric depression scale: a study of their validity for the diagnosis of a major depressive episode according to ICD-10 and DSM-IV. Int J Geriatr Psychiatry. 1999;14(10):858-65. Disponível em: https://doi.org/10.1002/(sici)10991166(199910)14:10\%3C858::aid-gps35\%3E3.0.co;2-8.

23. Kaiser MJ, Bauer JM, Ramsch C, Uter W, Guigoz Y, Cederholm T, et al. Validation of the Mini Nutritional Assessment Short-Form (MNA-SF): a practical tool for identification of nutritional status. J Nutr Health Aging. 2009;13(9):782-8. Disponível em: https://doi. org/10.1007/s12603-009-0214-7.

24. Miller MD, Paradis CF, Houck PR, Mazumdar S, Stack JA, Rifai AH, et al. Rating chronic medical illness burden in geropsychiatric practice and research: application of the Cumulative Illness Rating Scale. Psychiatry Res. 1992;41(3):237-48. Disponível em: https://doi.org/10.1016/0165-1781(92)90005-n.

25. Santiago LM, Luz LL, Mattos IE, Gobbens RJ. Cross-cultural adaptation of the Tilburg Frailty Indicator (TFI) for use in the Brazilian population. Cad Saúde Pública. 2012;28(9):1795-1801. Disponível em: https://doi.org/10.1590/S0102311X2012000900018.

26. Jerez-Roig J, Ferreira LMBM, Araújo JRT, Lima KC. Functional decline in nursing home residents: a prognostic study. PLoS ONE. 2017;12(5):e0177353. Disponível em: https://doi.org/10.1371/journal. pone. 0177353 .
27. Ferreira DB, Santiago LM, Mattos IE. The role of VES-13 as a predictor of adverse outcomes in elderly assisted by primary care, Brazil. Innov Aging. 2017;1(Supl 1):1-9. Disponível em: https://dx.doi. org/10.1093\%2Fgeroni\%2Figx004.4108.

28. Bull FC, Al-Ansari SS, Biddle S, Borodulin K, Buman M, Cardon G, et al. World Health Organization 2020 guidelines on physical activity and sedentary behavior. Br J Sports Med. 2020;54:1451-62. Disponível em: http://dx.doi.org/10.1136/bjsports-2020-102955.

29. Paterson DH, Warburton DE. Physical activity and functional limitations in older adults: a systematic review related to Canada's Physical Activity Guidelines. Int J Behav Nutr Phys Act. 2010;7(38):1-9. Disponível em: https://doi.org/10.1186/1479-5868-7-38.

30. Jonkman NH, Del Panta V, Hoekstra T, Colpo M, van Schoor NM, Bandinelli S, et al. Predicting trajectories of functional decline in 60- to 70-yearold people. Gerontology. 2018;64:212-21. Disponível em: https://doi.org/10.1159/000485135.

31. Boylu AA, Günay G. Life satisfaction and quality of life among the elderly: moderating effect of activities of daily living. Turk J Geriatr. 2017;20(1):619. Disponível em: https://www.researchgate.net/ publication/316062736_Life_satisfaction_and_ quality_of_life_among_the_elderly_Moderating_ effect_of_activities_of_daily_living.

32. Ng ST, Tey NP, Asadullah MN. What matters for life satisfaction among the oldest-old? Evidence from China. PLoS ONE.2017;12(2):e0171799. Disponível em: https://doi.org/10.1371/journal.pone.0171799.

33. Admi H, Shadmi E, Baruch H, Zisberg A. From Research to Reality: Minimizing the Effects of Hospitalization on Older Adults. Rambam Maimônides Med J. 2015;6(2):e0017. Disponível em: https://dx.doi.org/10.5041\%2FRMMJ.10201.

34. Osuna-Pozo CM, Ortiz-Alonso J, Vidán M, Ferreira $G$, Serra-Rexach JA. Review of functional impairment associated with acute illness in the elderly. Rev Esp Geriatr Gerontol. 2014;49(2):77-89. Disponível em: https://doi.org/10.1016/j.regg.2013.08.001.

35. Carvalho TC, Valle AP, Jacinto AF, Mayoral VFS, Boas PJFV. Impact of hospitalization on the functional capacity of the elderly: a cohort study. Rev Bras Geriatr Gerontol. 2018;21(2):134-42. Disponível em: https:// doi.org/10.1590/1981-22562018021.170143. 